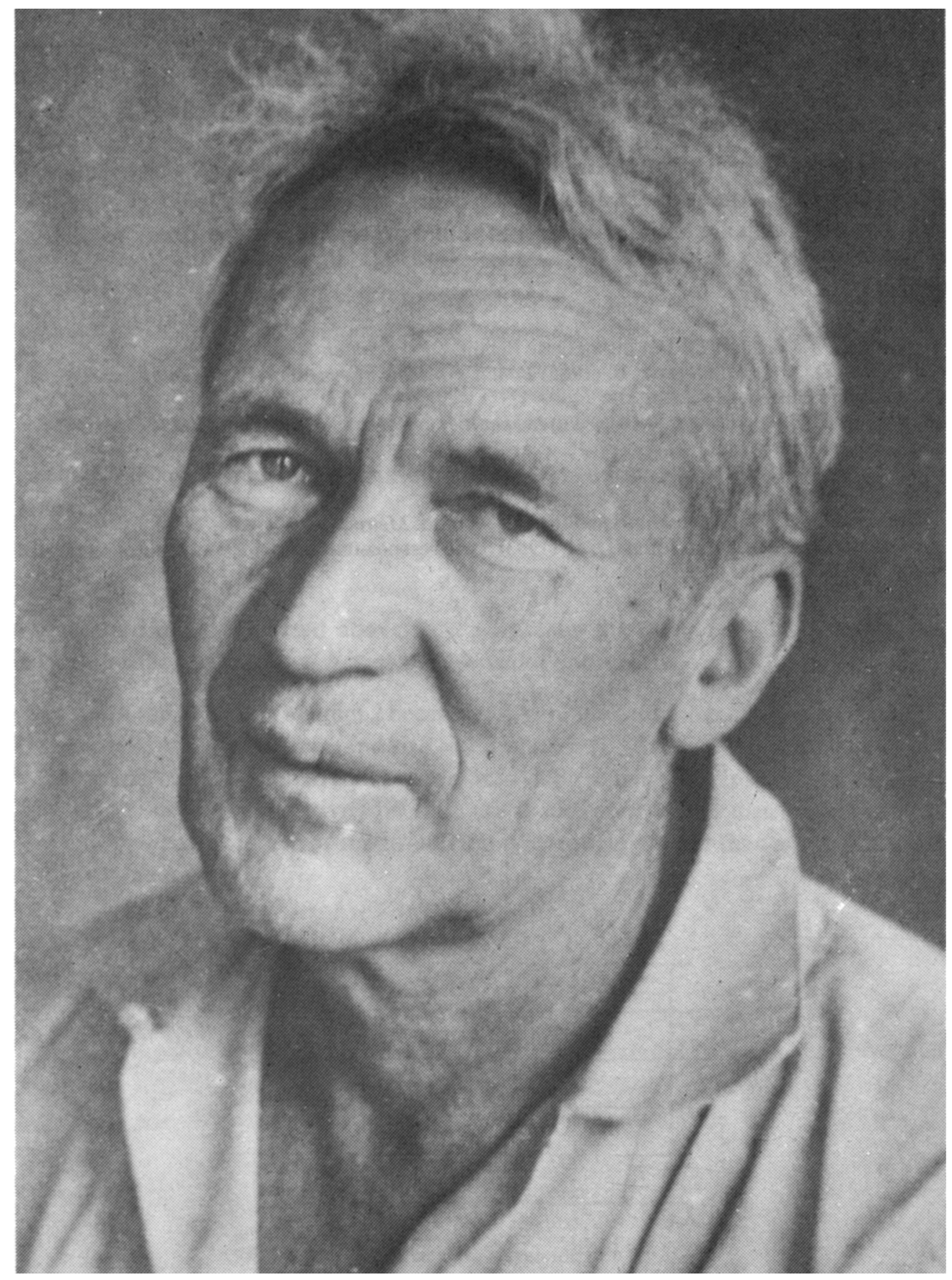

Andrei Nikolaevich Kolmogorov, 25 April 1903-20 October 1987 


\section{Obituary: ANDREI NIKOLAEVICH KOLMOGOROV}

Andrei Nikolaevich Kolmogorov, member of the USSR Academy of Sciences, one of the most eminent mathematicians of the twentieth century and the greatest probability theorist of recent times, died in Moscow on 20 October 1987 after a prolonged illness, at the ripe age of 84 . His life was one of intense mathematical creativity spanning six and a half decades and encompassing several disciplines. His achievement was outstanding, and he was presented with numerous honours and awards, including several honorary doctorates, foreign membership of many academies of science, among them the Royal Society of London, the National Academy of Sciences of the USA and the French Academy of Sciences, Paris. He was also the recipient of several orders of Lenin, and of the Balzan International prize in 1963; this he shared with Pope John XXIII, the historian S. Morrison, the chemist K. Frisch and the composer P. Hindemith.

Andrei Nikolaevich was born on 25 April 1903 in Tambov, where his mother Maria Yakovlevna was staying on a journey from the Crimea. Maria Yakovlevna died soon after the birth of her son, and the responsibility of nursing and subsequently educating Andrei fell on the shoulders of Maria's sister Vera Yakovlevna. On his mother's side Andrei Nikolaevich belonged to the nobility; his father was an agronomist. Andrei Nikolaevich was educated at Moscow University, where he subsequently taught as an instructor from 1925 until 1931; in that year he was appointed professor. He held the Moscow chair in the theory of probability from 1937 on.

Kolmogorov's creative ability in mathematics showed itself dramatically when, at the age of 19, he constructed an example of an $L_{1}$ function on the torus with a Fourier series whose partial sums $\left\{S_{n}(x), n=0,1,2, \cdots\right\}$ have the property $\lim \sup _{n \rightarrow \infty}\left|S_{2^{n}}(x)\right|=\infty$ almost everywhere. In his own words, this was discovered by him in the cabin of a running train while he was serving as its conductor during his university years. Till the end of his career he was an outdoor mathematician, to whom key ideas occurred while walking in the woods, swimming in the sea or in a lake, or skiing down the slopes of a mountain.

In 1933 his celebrated little book: Grundbegriffe der Wahrscheinlichkeitsrechnung (English translation: Foundations of the Theory of Probability, Chelsea 1950) appeared, in which an axiomatic approach to the theory of probability was outlined. To this day, it has stood the test of time and constitutes the cornerstone around which the entire edifice of statistical theory and computation is erected. The entire tapestry of the calculus of probability is woven around his ideas: these include the strong law of large numbers, conditional probability as a Radon-Nikodym derivative, the extension theorem for a consistent family of finite-dimensional distributions describing a stochastic process and 
a whole gamut of limit theorems for sums of independent random variables which are beautifully presented in his book with B. V. Gnedenko: Limit Distributions for Sums of Independent Random Variables (Addison-Wesley 1954; originally published in Russian 1949). The strong law of large numbers is at the heart of Monte Carlo methods in this computer age. Kolmogorov's inequality, from which the strong law was derived, is the forerunner of its more modern version in the avatar of Doob's inequality for semimartingales. The flavour of its proof lingers in the later successful attempts to unify the Birkhoff-Khinchine individual ergodic theorem and Doob's martingale convergence theorem. Kolmogorov's extension theorem has found wide applicability in mathematical logic, basic number theory and functional analysis. It inspired V. S. Varadarajan to deduce Riesz's representation theorem for continuous linear functionals on the Banach space $C(X)$ of continuous functions on a metric space, and to prove the existence of regular conditional probability distributions from the extension theorem. Bochner's theorem for positive definite functions, the Pontrjagin-Van Kampen duality theorem for locally compact abelian groups, the Gelfand-Neumark-Segal theorem for positive definite kernels, all have been derived from Kolmogorov's extension theorem. Kolmogorov's canonical form of the characteristic function of an infinitely divisible distribution with finite second moment was an intermediate step in the development of the famous Lévy-Khinchine formula which has found a variety of applications in areas like the theory of dams and collective risk theory.

In 1931, Kolmogorov wrote a fundamental paper entitled 'Über die analytische Methoden in der Wahrscheinlichkeitsrechnung' (Math. Ann. 104 (1931), 451-458) where he formulated the Chapman-Kolmogorov equation for the transition probabilities of a Markov process and derived the forward and backward equations for the transition probabilities of a diffusion process. To physicists these are known as FokkerPlanck equations. The problem of finding conditions for the existence and uniqueness of the transition probability satisfying a given Fokker-Planck equation was raised in this paper, and progress towards its solution continues to this day, starting from the seminal contribution of William Feller in 1936 and going through the tremendous flowering of the efforts of K. Ito, H. P. McKean, E. B. Dynkin, D. Stroock, S. R. S. Varadhan and several others. From mathematical genetics to demography and mathematical physics, there is no corner of applied probability left uninfluenced by developments following from Kolmogorov's 1931 paper.

Starting from the basic results of $\mathrm{H}$. Wold and A. Ya. Khinchine in the theory of stationary time series, Kolmogorov investigated the spectral properties of general weakly stationary stochastic processes. Independently of Norbert Wiener in the USA, he arrived at the linear prediction formula for the time-zero variable in terms of its past history. This has found a wide variety of applications in defence, meteorology and economics. The spectral approach to stationary processes led him to a deeper analysis of the correlation tensor of a homogeneous Gaussian random field over space-time describing the velocity field of a fluid in turbulence, and the discovery of the famous Kolmogorov spectrum and the equilibrium similarity law for fluids with low viscocity. In Kolmogorov's perception, the swelling corpus of information provided by linear combinations of observations on a stochastic process or a random field yields a filtration 
of subspaces of a Hilbert space or, equivalently, a spectral measure. If all measurable functions of observations are included in the analysis of a process, one obtains a filtration of $\sigma$-algebras. In the context of recent investigations in quantum probability, the same intuition leads to a filtration of $C^{*}$ or $W^{*}$ algebras. On the contemporary scene in statistics and probability, the idea of filtration of subspaces or $\sigma$-algebras originating from the Wiener-Kolmogorov approach is crucial in any dynamic analysis of data from which predictions and decisions are continuously revised on the basis of past experience.

More than a decade before the discovery of the more sophisticated methods of Ito's stochastic calculus, Kolmogorov derived the distribution of the maximum modulus of a Brownian path in a bounded interval by an ingenious repeated application of Bachelier's reflection principle. He also laid the foundation for non-parametric statistical inference by his creation of the famous Kolmogorov-Smirnov tests. His criterion for the continuity of sample paths of a stochastic process in terms of moments led his disciples to a more profound investigation of processes whose sample paths have discontinuities of the first kind only. His test for a positive continuous and increasing function on the halfline to belong to the upper or lower class with respect to the local continuity of Brownian motion has led to the study of the fine structure of Brownian motion.

Combining Shannon's ideas of a measure of information and information rate of a source, Kolmogorov extended their applicability to a domain far beyond the confines of information and coding theory. He created the Kolmogorov-Sinai entropy invariant for the classification of dynamical systems in 1958 (Dokl. Akad. Nauk SSSR (5) 119 (1958)) and thereby injected new life into ergodic theory, which was rather dormant after the discovery of von Neumann's discrete spectrum theorem. In this context, I cannot resist recalling a conversation I had with Professor S. Kakutani during one of his visits to the Mathematics Institute at the University of Warwick. Apparently, von Neumann suggested to Kakutani on his first visit to Princeton that he examine whether the notion of entropy could lead to a non-spectral invariant for the equivalence of measure-preserving transformations. This suggests a strong similarity in the thinking of two of the most eminent mathematicians of the century. Like von Neumann, Kolmogorov had an abiding interest in problems of logic, computational complexity and the randomness of numbers. The influence of Shannon's ideas led Kolmogorov to the formulation of $\varepsilon$ entropy and the Kolmogorov-Chaitin complexity of partial recursive functions. In the same vein, he investigated Hilbert's thirteenth problem and showed the existence of universal functions $f_{i j}, 1 \leqq i \leqq 7,1 \leqq j \leqq 3$ on the line which are real and continuous and such that every real continous function $\phi$ of three variables could be expressed as $\phi(x, y, z)=\sum_{i=1}^{7} g_{i}\left(f_{i 1}(x)+f_{i 2}(y)+f_{i 3}(z)\right)$ for some real continuous functions $g_{i}$, $1 \leqq i \leqq 7$. Soon after, his pupil V. I. Arnold solved Hilbert's thirteenth problem completely by setting it in the negative.

The interaction between probability theory, measure theory, topology and classical mechanics constituted one of the most passionate pursuits in the life of Kolmogorov. His greatest achievement in this direction is his initiation of the so-called KolmogorovArnold-Moser (KAM) theory which solved the long-standing problem of the stability of the solar system proposed by Henri Poincaré. In a celestial system consisting of only three bodies, say, the sun, the moon and the earth subject to the Newtonian law of 
gravitational attraction, if the moon is perturbed a little will it continue to remain in the system? The KAM theory shows that this is possible by proving the existence of quasiperiodic solutions of the $n$-body problem. As a spin-off from Kolmogorov's approach to the study of dynamical systems, we have the notion of $K$-automorphisms and $K$-flows which are fundamental to the study of chaos. The extension of this idea in the context of quantum theory is showing signs of fruitfulness thanks to the recent investigations of I. Prigogine, B. Misra and others. Sometimes mathematicians wonder why Kolmogorov did not pay attention to statistical questions that arise so naturally and profusely in quantum mechanics, especially when von Neumann's Mathematical Foundations of Quantum Mechanics had appeared as early as 1932 in German. My feeling is that we are already so much indebted to Kolmogorov for his contributions to mathematics in general, and probability theory in particular, that it is a blessing to have enough ground left for future mathematicians to till.

We, at the Indian Statistical Institute, cherish fond memories of Andrei Nikolaevich's visit to India in 1962 at the invitation of P. C. Mahalanobis and C. R. Rao. S. R. S. Varadhan and I were research scholars at the Research and Training School of the Institute at the time. I was assigned the difficult task of preparing a citation for Andrei Nikolaevich when the Institute, at a special convocation, conferred the degree of Doctor, Honoris Causa on him. Immediately after his arrival in Calcutta, Andrei Nikolaevich lost no time in plunging into discussions with the young students at the Institute about his recent research work on tables of random numbers, and the measurement of randomness of a sequence of numbers using ideas borrowed from mathematical logic. This piece of research was carried out by him during his travel by ship from the USSR to India; the ship was actually proceeding on an oceanographic expedition. Andrei Nikolaevich avoided air travel owing to some difficulty with his ears. He was a great swimmer, and according to him the warm sea voyage enabled him to plunge into the waters now and then for relaxation and the consequent stimulation of the brain. He went on to suggest that all institutes and universities in India should be located along the coastline of the peninsula, so that students and teachers could go for a swim before starting serious discussions.

When I explained to him my own modest results in information theory, he became quite enthusiastic and suggested to me the possibility of visiting the USSR. Mahalanobis, Rao and Kolmogorov worked out the bureaucratic details between them, and I was able to visit the Steklov Mathematical Institute and the University of Moscow during 1962-63.

Andrei Nikolaevich was a great humanist and he was visibly moved by the poverty and squalor which he saw in India, and particularly in Calcutta. During his walks in Indian towns he would stop here and there to take pictures of common people at work. He was very much puzzled by the intricate culinary procedures adopted at the roadside sweet shops, and took several pictures of ill-fed and ill-clad people energetically preparing dishes under the supervision of a manager. Later, in a lecture at the University of Moscow, he showed these pictures and made special reference to the coexistence of poverty and plenty in the same place.

Related to this was his interest in children and their education, particularly in 
mathematics. He founded a school for talented young mathematicians in Moscow and occasionally taught them, not only mathematics, but also music and literature. He also wrote and supervised the production of textbooks in algebra, analysis and geometry for Soviet schoolchildren.

To young research students he was easily accessible, and he showed great excitement when they made even a little progress on their own. However, to have the benefit of his valuable comments it was necessary to show enthusiasm for sport. Serious mathematics could be discussed with him while walking in the woods, rowing in a boat or relaxing after a swim. Indeed, key ideas for several of his results arose at various picturesque locations in the Soviet Union. I distinctly remember a couple of places in the countryside near Moscow where he stopped during our walks; he mentioned how the possibility of Prohorov's compactness theorem for a family of probability measures, and entropy as an invariant for a measure-preserving transformation, arose in those very places. Whenever young scholars happened to be in difficulty or in need of encouragement, he was ready to help them; I cannot resist narrating a few incidents that I personally experienced in this context. C. R. Rao, B. P. Adhikari, S. R. S. Varadhan and I, along with a few others, accompanied him on his journey to South India by train. We stopped in the town of Waltair where a seminar by Kolmogorov was organized in the Department of Mathematics and Statistics of Andhra University. We were all accommodated in comfortable rooms near the seashore. During the day we swam to our hearts' content, and later his seminar on stationary stochastic processes was held. Kolmogorov was welcomed by the professor of history, and thanked by the professor of law; the mathematicians were apparently ignored because they could not speak English with facility. Strangely enough Kolmogorov himself was not very familiar with the English language, and the seminar was delivered in French with a beautiful translation by Adhikari. Later in the evening the senior professors and Kolmogorov had dinner with the local minister of education, and the young guests were left out. The next morning Kolmogorov knocked on my door, came in and profusely apologized for participating in a dinner from which his young friends were excluded. During our travels we visited the shore temples of Mahabalipuram near Madras. I climbed the tower of one of the temples, slipped, fell down from the top, fractured a few bones in my right foot and landed in a city hospital with the assistance of Varadhan. Later in the evening Kolmogorov visited me in the hospital, looked at my injured foot and remarked: 'Biological systems are highly stable, you are young and you will be up in no time. Don't worry.'

I should like to recall another incident that occurred during my stay in Moscow. I was invited to spend a day at his dacha where he was living with Professor P. S. Alexandrov, the well-known topologist. A. Shiryaev and V. V. Sazonov accompanied me on the train journey from Moscow. Andrei Nikolaevich met us one stop ahead of the scheduled one, gave me pair of robust shoes to wear and suggested a walk in the woods in deep snow. The precarious walk of a frail Indian in oversize shoes tramping in deep snow provided my Russian friends with a lot of fun. Very soon Andrei Nikolaevich plunged into mathematical conversations, as was his habit on such occasions. He got excited about the complexity of different organisms and how they could be compared in a mathematically meaningful way. Is it possible to compare the complexity of different civilizations 
by looking at the structure of their languages or art? I was a quiet listener. After a rather long walk we reached his dacha, where we were welcomed by P. S. Alexandrov with the following words: 'I know two Indian mathematicians, the famous number theorist Ramanujan and a fine topologist Vaidyanathaswamy (which he pronounced with considerable difficulty).' I had plenty of vegetarian food followed by fine classical music which went on till late in the evening. On our journey back to Moscow, I was unexpectedly held up by a policeman at the local railway station and asked to produce my documents, which I had left at my hotel. Meanwhile the train arrived, and somehow Shiryaev managed to push me into a carriage and jumped in after me. The next morning at the University, Andrei Nikolaevich himself appeared and apologised for the unpleasant incident the previous night at the railway station. He recalled an incident when he himself was held up by the police: they found a gold spoon in his pocket but no papers to establish his identity.

Kolmogorov inspired many generations of students whose list includes such eminent mathematicians as I. M. Gelfand, V. I. Arnold, Ya. G. Sinai, E. B. Dynkin, Yu. V. Prohorov, R. L. Dobrushin, B. V. Gnedenko, A. Rényi and many others. The community of mathematicians in general, and probability theorists in particular, owe him a great debt for the legacy of his work. This is being published in a series of volumes by the USSR Academy of Sciences. It is hoped that an English translation of these volumes will soon appear, to educate and inspire future generations of mathematicians all over the world. We can then admire the landscape of mathematics as a whole, without becoming lost in a maze of isolated results.

In conclusion, it may be fitting to quote the statement made by the American statistician J. Wolfowitz, at the 1963 All-Union Conference of Probability and Statistics in Tbilisi: 'I came to the USSR with the specific purpose of finding out whether Andrei Nikolaevich Kolmogorov is an individual or an institution.'

Indian Statistical Institute

K. R. PARTHASARATHY

Delhi

February 1988 